\title{
ANALYSIS ON PARAMETERS AFFECTING SLEEP APNEA \& CONTROL MEASURES USING LOW COST POSITIVE AIR PRESSURE SUPPLY MACHINE
}

\author{
Sandeep Reddy AV \\ Assistant Professor, Dept of BME, Gokaraju Rangaraju Institute of Engineering \& Technology, Hyderabad
}

\begin{abstract}
Sleep apnea is a potentially serious sleep disorder in which breathing repeatedly stops and starts for at least ten seconds. Apnea may occur 5 to 30 times or more in patients during sleep. Sleep apnea is diagnosed with an overnight sleep test called a polysomnogram, or "sleep study". More than eighteen million Americans suffer from sleep apnea (ASAA), and it is estimated conservatively that ten million remain undiagnosed. There are many ways to control sleep apnea i.e., through respiratory devices (CPAP, Bi-PAP), surgery on mouth and throat, Oral Appliance Therapy (OAT) etc. These respiratory devices are not self controlled which disturbs the patients sleep and should be turn ON throughout the night continuously
\end{abstract}

In this Research, the objective is to develop a Bi-PAP device (Automatic Bi-level Positive Airway Pressure machine) for the purpose of detection and controlling of sleep apnea disease using Atmel 89c51 Microcontroller programming. Here, a new methodology is followed to detect the sleep apnea condition in a patient by using active Infrared source and Infrared detector. The program is specifically to control the functioning of the Bi-PAP and is shown to be effective at responding to a patient's breathing cycle. Central sleep apnea can also be detected and alarmed such that this ability could prove critic during emergency situations.

In particular, the current Bi-PAP machine operates depending up on the patient's necessity for positive air pressure. Therefore the BiPAP acting time on a patient can be reduced and hence, BiPAP side effects can be minimized. A Humidifier is also engaged along with saturated oxygen line to clear patients' airway. Based on the results, the Automatic BiPAP system is shown to be more effective at responding sleep apnea condition by giving alarm signal. It is economically good and maintenance free. Exclusively, in this system Apneas both in infants and adults are detected by the adjustable sensing time. Current design acts positively on demerits of existing methodologies. The system has been validated on a single live subject in the Sleep Research Laboratory. Finally, it is found that this fully functional Bi-PAP is compatible for both home \& hospital usage. Development of this device in several aspects will be a great achievement.

***

\section{1: INTRODUCTION}

\subsection{Sleep}

Sleep is a naturally recurring altered state of consciousness with relatively suspended sensory and motor activity, characterized by the inactivity of nearly all voluntary muscles. We know that our brains are very active during sleep. Moreover, sleep affects our daily functioning and our physical and mental health in many ways.

During sleep, we usually pass through five phases of sleep: stages 1, 2, 3, 4 and REM (Rapid Eye Movement) sleep fig 1.1. These stages progress in a cycle from stage 1 to REM sleep, then the cycle starts over again with stage 1 .We spend almost 50 percent of our total sleep time in stage 2 sleep, about 20 percent in REM sleep, and the remaining 30 percent in the other stages. Infants, by contrast, spend about half of their sleep time in REM sleep.

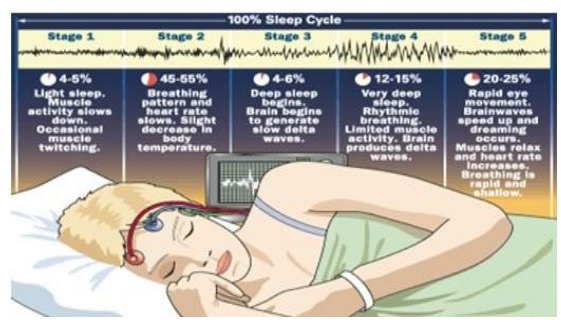

Fig 1.1 Sleep Cycle of a Healthy Subject

Sleep appears necessary for our nervous systems to work properly. Too much of little sleep leaves us drowsy and unable to concentrate the next day. It also leads to impaired memory and physical performance and reduced ability to carry out math calculations. If sleep deprivation continues, hallucinations and mood swings may develop. Without sleep, neurons may become so depleted in energy or so polluted with byproducts of normal cellular activities that they begin to malfunction. 
Sleep also may give the brain a chance to exercise important neuronal connections that might otherwise deteriorate from lack of activity. The most common sleep disorders include insomnia, sleep apnea, restless legs syndrome, and narcolepsy.

\subsection{Sleep Apnea}

Sleep apnea is a common sleep disorder characterized by brief interruptions of breathing during sleep."Apnea" is a Greek word meaning "without breath." An apnea is clinically defined as a cessation (pause) of breath that lasts at least ten seconds. Apnea may occur 5 to 30 times or more in patients during sleep. Similarly, each abnormally low breathing event is called a hypopnea. Sleep apnea is diagnosed with an overnight sleep test called a polysomnogram, or "sleep study". More than eighteen million Americans suffer from sleep apnea (ASAA), and it is estimated conservatively that ten million remain undiagnosed.

\section{Other Names for Sleep Apnea}

- Cheyne-Stokes breathing

- Sleep-disordered breathing

There are three forms of sleep apnea: central (CSA), obstructive (OSA), and complex or mixed sleep apnea (i.e., a combination of central and obstructive) constituting $0.4 \%, 84 \%$ and $15 \%$ of cases respectively.

In CSA, Central sleep apnea, which occurs when patient's brain doesn't send proper signals to the muscles that control breathing; In OSA, Obstructive sleep apnea breathing is interrupted by a physical block to airflow despite respiratory effort, and snoring is common. Regardless of type, an individual with sleep apnea is rarely aware of having difficulty breathing, even upon awakening. Symptoms may be present for years (or even decades) without identification, during which time the sufferer may become conditioned to the daytime sleepiness and fatigue associated with significant levels of sleep disturbance.

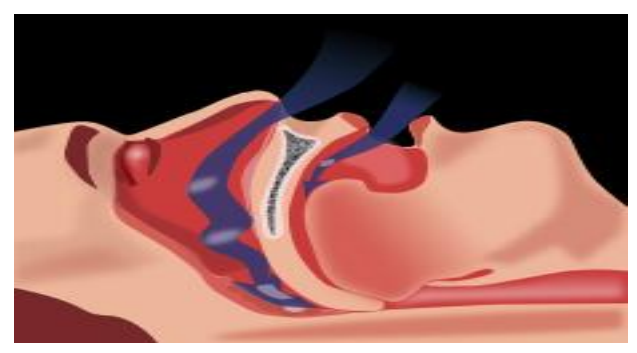

Fig.1.2 During sleep apnea, upper airway completely blocked

Typically the soft tissue in the rear of the throat collapses and closes the airway, forcing victims of sleep apnea to stop breathing repeatedly during sleep, as frequently as a hundred. Although the typical sleep apnea patient is overweight, male and over the age of forty, sleep apnea affects both males and females of all ages and of ideal weight.

\subsubsection{Risk Factors for Sleep Apnea}

- A family history of sleep apnea

- Excess weight

- Being older

- A narrowed airway or tonsils: Obstructive sleep apnea (OSA) is common among children with enlarged tonsils or adenoids.

- High blood pressure (hypertension)

- A large neck(fat)

- A recessed chin

- Abnormalities in the structure of the upper airway

- Smoking \& Alcohol usage

\subsubsection{Symptoms}

The signs and symptoms of obstructive and central sleep apneas overlap, sometimes making the type of sleep apnea more difficult to determine. The most common signs and symptoms of obstructive and central sleep apneas include:

- Loud snoring, which is usually more prominent in obstructive sleep apnea

- Excessive daytime sleepiness (hypersomnia).

- Observed episodes of breathing cessation during sleep.

- High BP \& other cardiovascular complications.

- Morning headaches.

- Abrupt awakenings accompanied by shortness of breath, which more likely indicates central sleep apnea.

- Impotence.

- Memory problems.

- Feelings of depression.

- Reflux.

- Nocturia.

- Awakening with a dry mouth or sore throat.

- Difficulty staying asleep (insomnia)

\subsection{Tests and Diagnosis}

Based on patient's signs and symptoms involving overnight monitoring of breathing and other body functions during sleep a sleep specialist will decide need for further evaluation. Tests to detect sleep apnea may include:

Nocturnal polysomnography or sleep study : During this test, patient is hooked up to equipment that monitors heart rate and rhythm (ECG), lung and brain activity (EEG), breathing patterns using oral and nasal sensors, arm and leg movements (EMG), Eye and Chin movement (EOG) and blood oxygen levels while you sleep (OXIMETRY).

\subsubsection{Treatments for Sleep Apnea}

- Avoidance of alcohol

- Medications that relax the airway and/or reduce respiratory drive.

- Wearing an oral appliance designed to keep patient's throat open. 
- Surgery removing excess tissue from nose or throat that may be blocking patient's upper air passages and causing sleep apnea.

- $\quad$ Lose excess weight.

- Avoid certain medications such as tranquilizers and sleeping pills.

- Positional therapy: Sleep on patient's side or abdomen rather than on patients back.

- Keep patients nasal passages open at night.

- CPAP or Bi-PAP usage.

- Adaptive servo-ventilation (ASV) and Supplemental oxygen in case of central and complex sleep apnea.

\section{Untreated sleep apnea can:}

- Increase the risk of high blood pressure, heart attack, stroke, obesity, and diabetes

- Increase the risk of, or worsen, heart failure

- Make arrhythmias (ah-RITH-me-ahs), or irregular heartbeats, more likely

- Increase the chance of having work-related or driving accidents

\subsection{Treatment}

Some treatments involve lifestyle changes such as avoiding alcohol or muscle relaxants, losing weight, and quitting smoking. Many people benefit from sleeping at a 30-degree elevation of the upper body or higher. Doing so helps prevent the gravitational collapse of the airway i.e., prevent the tongue and palate from falling backwards in the throat and blocking the airway. Lateral positions (sleeping on a side), as opposed to supine positions (sleeping on the back) are also recommended as a treatment for sleep apnea largely because the gravitational component is smaller in the lateral position. There are surgical procedures to remove and tighten tissue and widen the airway. Some people benefit from various kinds of oral appliances to keep the airway open during sleep. Continuous positive airway pressure (CPAP or BiPAP) is the most effective.

For moderate to severe sleep apnea, the most common treatment is the use of a continuous positive airway pressure (CPAP) or Bi-level Positive Airway Pressure (BiPAP) device, which 'splints' the patient's airway open during sleep by means of a flow of pressurized air into the throat. The patient typically wears a plastic facial mask, which is connected by a flexible tube to a small bedside CPAP or BiPAP machine. The BiPAP machine generates the required air pressure to keep the patient's airways open during sleep. Advanced models may warm or humidify the air and monitor the patient's breathing to ensure proper treatment. BiPAP therapy is extremely effective in reducing apneas and less expensive than other treatments, but some patients find it extremely uncomfortable and refuse to continue the therapy or fail to use their BiPAP machines on a nightly basis.
In addition to BiPAP, dentists specializing in sleep disorders can prescribe Oral Appliance Therapy (OAT). The oral appliance is a custom-made mouthpiece that shifts the lower jaw forward, opening up the airway. OAT is usually successful in patients with mild to moderate obstructive sleep apnea.

Several levels of obstruction may be addressed in physical treatment, including the nasal passage, throat (pharynx), base of tongue, and facial skeleton. Surgical treatment for obstructive sleep apnea needs to be individualized in order to address all anatomical areas of obstruction. Often, correction of the nasal passages needs to be performed in addition to correction of the oropharynx passage. Septoplasty and turbinate surgery may improve the nasal airway.

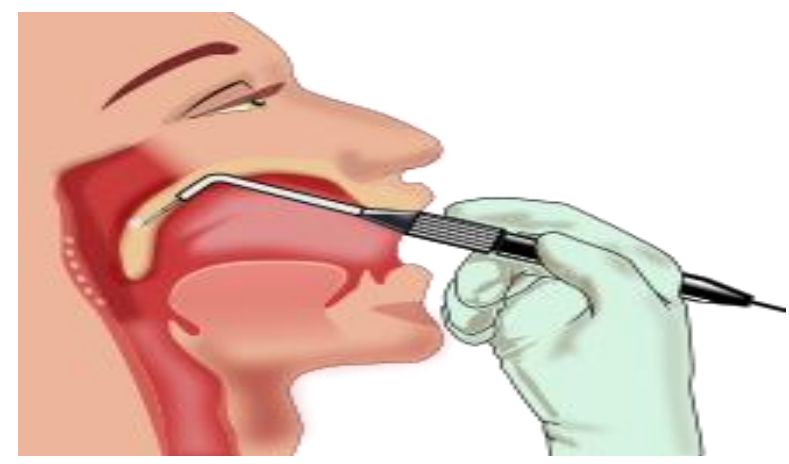

Fig 1.3. Illustration of surgery on the mouth and throat

Other surgery options may attempt to shrink or stiffen excess tissue in the mouth or throat, procedures done at either a doctor's office or a hospital. Small shots or other treatments, sometimes in a series, are used for shrinkage, while the insertion of a small piece of stiff plastic is used in the case of surgery whose goal is to stiffen tissues. Surgery on the mouth and throat, as well as dental surgery and procedures, can result in postoperative swelling of the lining of the mouth and other areas that affect the airway.

In case of OSA, Not enough air flows into patient's lungs if patient's airway is fully or partly blocked during sleep. This can cause loud snoring and a drop in patients' blood oxygen level. If the oxygen drops to a dangerous level, it triggers patient's brain to disturb patients sleep. This helps tighten the upper airway muscles and open patient's windpipe. Normal breaths then start again, often with a loud snort or choking sound.

The frequent drops in oxygen level and reduced sleep quality trigger the release of stress hormones. These compounds raise patient's heart rate and increase patient's risk of high blood pressure, heart attack, stroke, and arrhythmias (irregular heartbeats). The hormones also raise the risk of, or worsen; heart failure. Untreated sleep apnea also can lead to changes in how patient's body uses energy. These changes increase patient's risk of obesity and diabetes. 


\subsection{Basic Idea}

The main idea behind developing this research is evolved from a bio-medical design of CPAP device [2]. As per this design CPAP device is used to Nebulized drug delivery to the sleep apnea patient automatically. The exact preview of the designs is shown below.

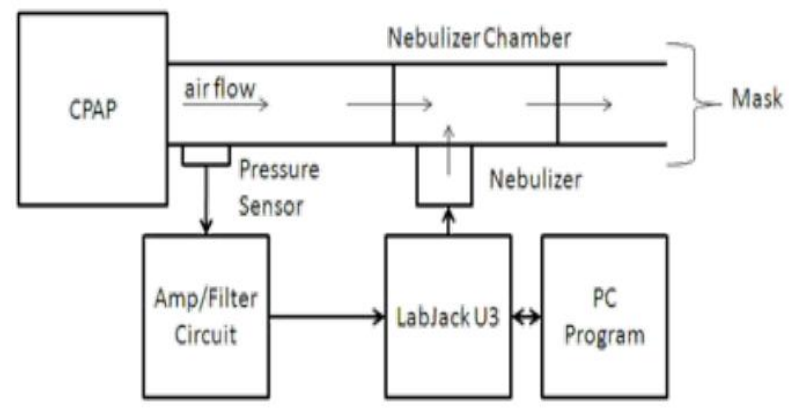

Fig.1.4 Nebulized Drug Delivery through Continuous Positive Airway Pressure Device.

Generally, a CPAP device is prescribed to a patient when he/she is unable to take breathing normally. In specific, Patients with Obstructive Sleep Apnea (OSA) disease uses CPAP device to eliminate pausing breaths automatically. The CPAP/BIPAP Machines using in present days does not have option to switch $\mathrm{OFF} / \mathrm{ON}$ automatically depending on patients necessity. Besides patients' requirement, he/she should wear the device continuously whole night.

A Method is introduced for Automated Delivery of Respiratory Bronchodilator Medications like albuterol, to Sleep Apnea patients as they use the CPAP device [30, 31].

Continuous Positive Airway Pressure (CPAP) Devices

- Include a pressure source (blower), a circuit, and tubing to a mask worn by a patient.

- Provide positive pressure to assist in patients breathing.

- Used during sleep or in ambulatory situations for patients with sleep apnea or asthma.

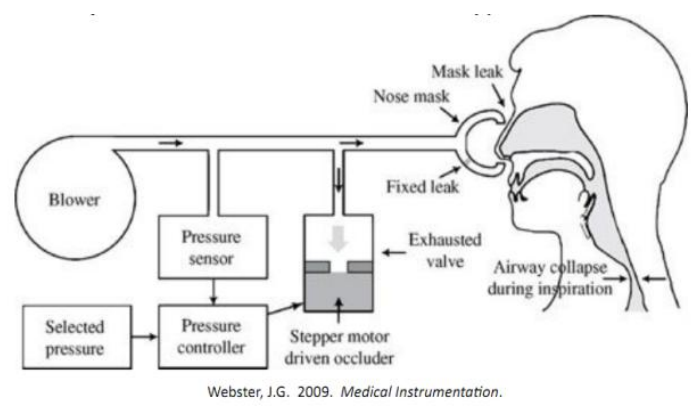

Fig.1.5 Internal Components in a CPAP device

\section{LITERATURE SURVEY}

\subsection{Theoretical Analysis on Sleep Apnea}

As per T. Penzel [1] explanation, Sleep disorders have a high prevalence. Sleep disorders are recognized first by the complaint of non restorative sleep. Some particular sleep disorders such as sleep apnea are characterized by many very short awakenings. These very short awakenings are called arousals. The arousals disrupt sleep. This sleep fragmentation can cause severe daytime sleepiness. The daytime sleepiness is dangerous because it can lead to unwanted falling asleep at monotonous tasks as car driving or complex system supervision (e.g. power plant controls). A quantification of the disorder is done by the investigation in a sleep laboratory. The polysomnography investigation in the sleep laboratory examines the EEG, EOG and EMG to derive sleep stages or scoring. Figure below indicates sleeping cycle of a patient such that in the beginning of the night there is more deep sleep and in the morning there is more REM sleep.

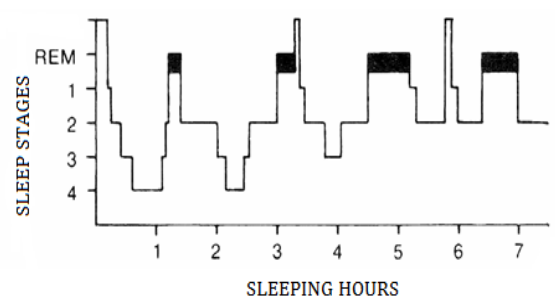

Fig.2.1. The Normal Distribution of Sleep Stages Over the Night.

Usually the time course of the sleep stages is quantified in terms of percentages of stages related to total sleep time and the latencies for the individual stages. The additional feature of transitions between sleep stages and the disruption of sleep, which corresponds to periods of wakefulness during sleep, are evaluated systematically [1].Usually sleep fragmentation is quantified in terms of simple statistics such as the number of arousals, of awakenings, the relative time of wakefulness during sleep related to the time spent in bed (sleep efficiency).

The basic aim in this article [1] was to characterize the sleep fragmentation using a new measure. This new measure was enabled to differentiate disturbed sleep and normal sleep in a better way than just giving percentages of sleep stages. This measure can differentiate the normal sleep of healthy subjects and the fragmented sleep of sleep apnea subjects. It was helpful to quantify age effects on sleep and sleep stages as well as other pathologies (such as narcolepsy, insomnia, depression and dementia) which also influence sleep very much.

Obstructive sleep apnea syndrome is highly prevalent in adult population and increases cardiovascular morbidity and mortality according to Daniela Boisteanu [3]. Nasal continuous positive airway pressure (CPAP) remains the treatment of choice for 
obstructive sleep apnea (OSA). Automatic CPAP (APAP) can be use, not only for treatment but also for suggesting the optimum CPAP setting. Ninety-five newly diagnosed patients were included (mean age 51 \pm 9.6 years) with moderate and severe obstructive sleep apnea syndrome.

The patients were randomly allocated to one of the investigated devices: APAP or fixed pressure CPAP. A lower mean effective pressure seen in AUTO group $7.67 \mathrm{~cm} \mathrm{H} 2 \mathrm{O}$ vs. CPAP $8.51 \mathrm{~cm}$ $\mathrm{H} 2 \mathrm{O}$ and CPAP patients presented statistically significant lower residual AHI. As a conclusion the AUTO approach seems to be similarly effective as CPAP and provide a method for determining a single fixed pressure, suitable for long term treatment with a conventional CPAP device.

In certain apnea detection methodology, Fu-Chung Yen [4] pharyngeal wall vibration (PWV) signal is collected and sent through feedback to detect the OSA condition. Two positively diagnosed sleep apnea patients are engaged in this study

A computer-based data acquisition system was used to collect the APAP pressure signal during the sleeping hours of the two patients. The APAP therapy concludes the pressure drop occurring in comparing pressure signals of the patients. Therefore, adjusting the blower pressure automatically based on PWV signal detection may provide comparable treatment to that of conventional CPAP device with lower airway pressure.

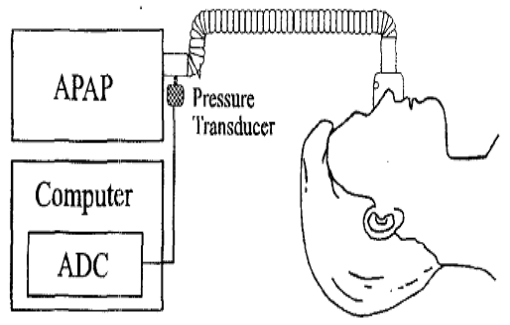

Fig.2.2 Configuration of flow through Automatic Continuous Positive Airway Pressure

The Auto-CPAP as discussed Richard B. Berry [5] is to estimate the pressure level required for an Obstructive Sleep Apnea patient, based on an approach. That is to determine the required level of CPAP during a trial (titration) in the sleep laboratory using an attended study with complete EEG and respiratory monitoring. In general, higher pressure is needed in the supine position or during rapid eye movement (REM sleep). Problem with conventional fixed CPAP treatment was that a pressure sufficient to maintain upper airway patency in all situations must he used for the entire night. This pressure may be appreciably higher than needed for major portions of the night. Auto CPAP (APAP) can provide a solution to some of the above problems. The devices monitor some or all of the following variables: flow (apnea or hypopnea), snoring (airway vibration), airflow profile flattening (airflow limitation), or airway impedance (forced oscillation technique).
Depending on the monitored variables, Pressure is slowly increased until apnea; hypopnea, arterial oxygen desaturation, snoring, and respiratory effort related arousals are prevented in all body positions and sleep stages. Each APAP device has proprietary algorithms to slowly increase pressure in response to the detected respiratory events. Once all events are eliminated, pressure is slowly reduced until events are again detected. In this manner the lowest effective pressure is provided (autoadjusting). The APAP units also contain memory that stores time at pressure and detected event information. The stored information can be quickly transferred to a computer for analysis. One can determine the average, maximum, and 95th percentile pressure (P95) over one or several nights. This allows selection of a fixed pressure for conventional treatment with fixed pressure CPAP (auto-titration).

There is a methodology for controlling or treating sleep apnea syndrome by employing air-bag actuation according to Shunji Moromugi [6]. We know that Airway of OSA patient is frequently obstructed during sleep because of tongue swallowing. This apparatus is composed of a pneumatically actuated flexible vest and a controller including an air pressure control unit. The flexible vest has sensors to detect airway obstructions and two airbags to conduct head tilt -chin lift maneuvers that are needed for airway establishment. The proposed treatment apparatus successfully detects the airway obstruction and automatically establish the airway by conducting the chin lift-head tilt maneuvers.

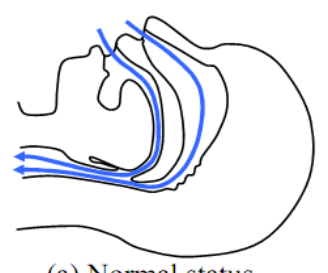

(a) Normal status

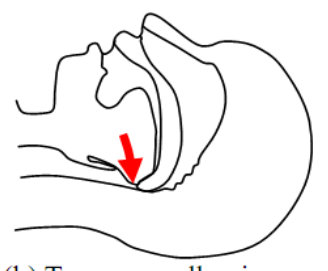

(b) Tongue swallowing
Fig. 2.3 Upper airway closure by tongue swallowing in OSA patients

\section{Working procedure:}

There are two manual operations to effectively prevent the tongue swallowing. One is head-tilt Controller is composed of a microcomputer, an air pressure control unit, and two air pressure sensor Microcomputer judges whether the user's airway is obstructed or not. Once airway obstruction is detected then microcomputer sends control signals to the air pressure control unit to inlet air to airbags so that the airbags inflate and head tilt-chin lift maneuvers are conducted. The air is gradually exhausted and the vest gets back to initial shape after observing normal aspiration for a specific period. 


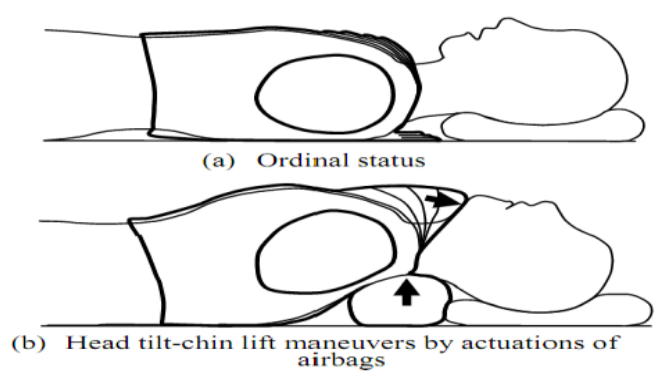

Fig.2.4 Mechanism of the vest actuation to open airway

There is an approach based on the finding that OSA frequently alters healthy heart rate dynamics by JE Mietus [7]. In normal healthy respiration, heart rate dynamics exhibit a broadband, inverse power law spectral distribution. In contrast, during periods of prolonged OSA, the heart rate typically shows cyclic increases and decreases associated with the apneic phase and the resumption of breathing. These cycles which tend to oscillate at a frequency of between 0.01 and $0.04 \mathrm{~Hz}$, are a distinctive feature of OSA not found during normal respiration. They hypothesized that it could detect and quantify these periods of high-density OSA by the fully automated identification of these oscillatory dynamics in the RR inter beat interval series.

Apnea can be detected by analyzing Hilbert transform amplitude and frequency probability distributions for the means, standard deviations and time within threshold limits for the 5minute windows of all periods of normal respiration and highdensity OSA Parameter limits.
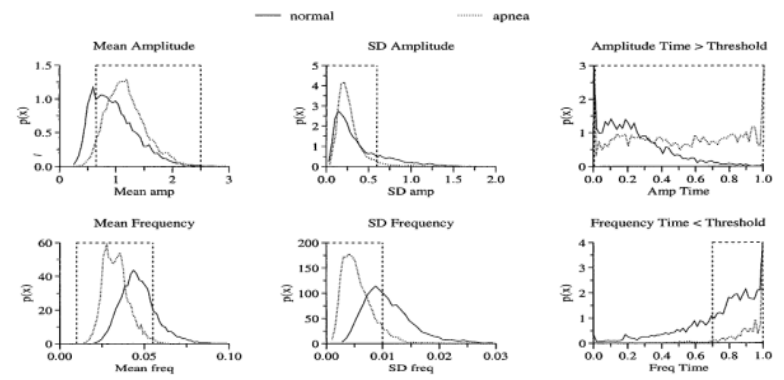

Fig.2.5 Parameters varying for Normal and Abnormal (apnea) intervals

In a practical survey, (sleep laboratory - Bhagwaan Mahaveer Jain Multi Specialty hospital, Bangalore) I came across different methodologies in detecting sleep apnea during the whole night (complete sleep study) of a patient. These methodologies are as given below.

- $\quad$ EEG - through monitoring brain waves

- $\quad$ EOG - eye and chin movement

- ECG - heart rate \& rhythm

- Nasal \& Oral flow sensor

- $\quad \mathrm{EMG}$ - Muscle movement near abdomen
- Additional monitors like pulse-Oximeter to sense $\mathrm{O}_{2}$ and $\mathrm{CO}_{2}$ in blood

A clear report of a patient by checking the above parameters gives an idea that a sleep apnea patient initially (at early stages of sleep) does not come across apnea. After or later certain time of sleep cycle, the muscle relaxation (closure of upper airway) takes place leading to apnea. These apneas continue to occur disturbing the patient entire sleep cycle. In case of Obstructive Sleep Apnea, The patient receives a signal from brain which disturbs sleep stage. As this continues during the whole night he/she faces side effects and diseases as discussed in previous chapter (sleep apnea-introduction).

In accordance with Ashida Nobuyuki [8], it has been pointed out that sleep of those who have apnea attacks is characterized by snoring in many cases, and thus attempts have been made to conduct screening for sleep apnea syndrome by analyzing snoring sounds. However, using snoring sound for screening is not practical at present because enormous effort is required in order to analyze each individual's overnight snoring sound for a clinical application purpose. As just described, screening for sleep apnea syndrome has been regarded as being considerably troublesome. In addition, snoring sound with relatively less noise can be collected in well-equipped environments such as a sleep disorder center, but, collecting snoring sound at home suitable for analysis is likely to be difficult because numerous different kinds of noise sources exist at home.

With intent to improve such situations, use of an IC recorder with a bone-conduction microphone and a simple $\mathrm{SpO}_{2}$ monitor was considered and sound processing was used to eliminate noise in this study this time with expectation that such application of those devices will minimize patients' psychological burdens because staying overnight at hospital to undergo an examination in an extensively-equipped room will become unnecessary. As reduction of $\mathrm{SpO}_{2}$ during sleep results in sleep quality deterioration, it was studied if comparison between the $\mathrm{SpO}_{2}$ and pulse data and the snoring sound data, both of which were collected during sleep, could be helpful in order to grasp respiratory status during sleep \& evaluate sleep quality, using the main symptom of sleep apnea syndrome, snoring.

As per David P. Kuehn [9], therapeutic procedures could be effective in reducing hyper-nasality because the availability and use of newer instrumentation such as nasoendoscopy which is capable of providing feedback to subjects during speech. Feedback procedures eventually may be shown to be effective in reducing hyper nasality, although data are needed.

Kuehn [9] proposed a resistance type of treatment for strengthening the muscles of velopharyngeal closure that utilizes well-known principles of exercise physiology. Resistance to the muscles is provided by delivering heightened air pressure to the nasal cavities using a commercially available 
device referred to generically as continuous positive airway pressure (CPAP). Major principles of the treatment include overloading, progressive resistance, and specificity of the exercise regimen, all of which are known to increase muscle strength. The greater the overload, the greater the strength gains that can be expected for the muscle or muscle group being exercised. If overloading does not occur, further increases in strength will not be obtained. This is an important consideration with regard to the muscles of velopharyngeal closure because Non-speech activities, such as swallowing and blowing, still are frequently used in an attempt to strengthen the muscles. Thus, it is advantageous to provide resistance to the muscles of velopharyngeal closure during speech individuals with marginal velopharyngeal inadequacy, in particular, may tend to elevate the velum to a position involving muscle activity.

This would tend to avoid a Situation in which fatigue occurs and velar elevation becomes very difficult or impossible resulting in very severe hyper-nasality. The CPAP therapy proposed by Kuehn could potentially increase both muscle endurance as well as strength because it overloads the levator veli palatini muscle and involves a regimen with a large number of repetitions of velar elevation.

According to $T$. Penzel [13], a reliable diagnosis with relatively simple and portable methods is needed. One approach is to make use of autonomous nervous system changes which accompany disordered breathing during sleep. The peripheral arterial tonometry (PAT) determines the peripheral arterial vascular tone using a plethysmographic method on the finger. The peripheral arterial tone is modulated by sympathetic activity, by peripheral blood pressure, and by the peripheral resistance of the vessels. He investigated a new ambulatory recording device which uses PAT, oximetry and actigraphy in order to detect sleep apnea. The ambulatory recording system (Watch-PAT 100) consists of two finger sensors for the PAT and for the pulse oximetry. The sensors are connected to the amplification unit. This unit contains the actigraphy and the flash memory card and the rechargeable batteries.

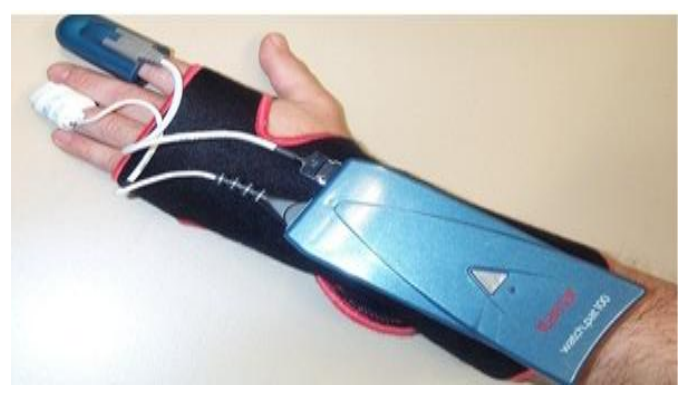

Fig 2.6 shows ambulatory recording system consisting of two finger sensors for the peripheral arterial tonometry and for the pulse oximetry

For this purpose he performed a comparative study on 21 patients referred to sleep laboratory due to suspected sleep apnea. The Watch-PAT was used in parallel with cardio respiratory polysomnography and the validity was determined. The new system was able to detect apneas and hypopneas with a high reliability $(\mathrm{r}=0.89)$. It has sensitive to arousals $(\mathrm{r}=0.77)$. As per this article, it was concluded that the system was very well suited to perform control studies in patients with sleep apnea which are under therapy and require regular follow-up investigations to maintain a high CPAP compliance.

As said by Alfredo Burgos [20], The SAMON system was provided with a very accurate classifier which has capability of identifying the presence of sleep apneas from blood oxygen saturation signal fragments taken from pulsioximetry systems (SpO2). He divided the $\mathrm{SpO} 2$ signals of Apnea-ECG into fragments of 60 seconds and then was preprocessed on every $\mathrm{SpO} 2$ signal fragment to extract relevant features from the signal: the odi indexes and the tsa indexes [20]. A set of experiments were performed with the Weka tool over the previous data set. Weka tool splits $\mathrm{S}$ into two sets: the training data set and the test data set. The best classification model was the meta-classifier Bagging which uses the (decision tree) ADTree as base classifier.

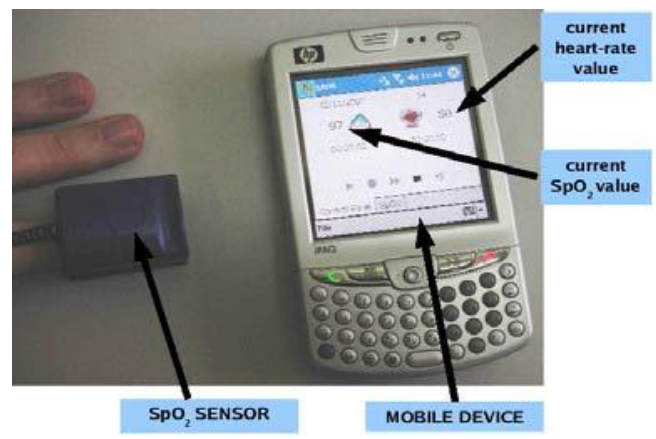

Fig.2.7 shows sleep apnea monitoring with portable oximetry device

SAMON is an apnea monitoring system able of, on the one hand, recording nocturnal oximetry and sending it to the hospital so that it can be analyzed by pneumologists with the same analyzing software they use daily at their hospitals and, on the other hand, identifying in real-time the presence of desaturations related to apneic events by using apnea classifier. Therefore, the system was considered as a clinical decision support system to aid pneumologists to diagnose apnea.

Currently, there is no economically free device which can automatically sense sleep apnea and which can control its effect without disturbing sleep state of a patient. In case of current research (Detection and Control of Sleep Apnea through Automatic BiPAP device), An explicit new methodology is followed in order to detect the sleep apnea condition in a patient by using active Infrared source and detector sensor. A fully controlled Microcontroller circuit is programmed to switch ON/OFF the BiPAP machine automatically such that the sleep 
state will not be disturbed .This concept is discussed in detail in upcoming chapters.

\section{CURRENT METHODOLOGY}

\subsection{Block Diagram}

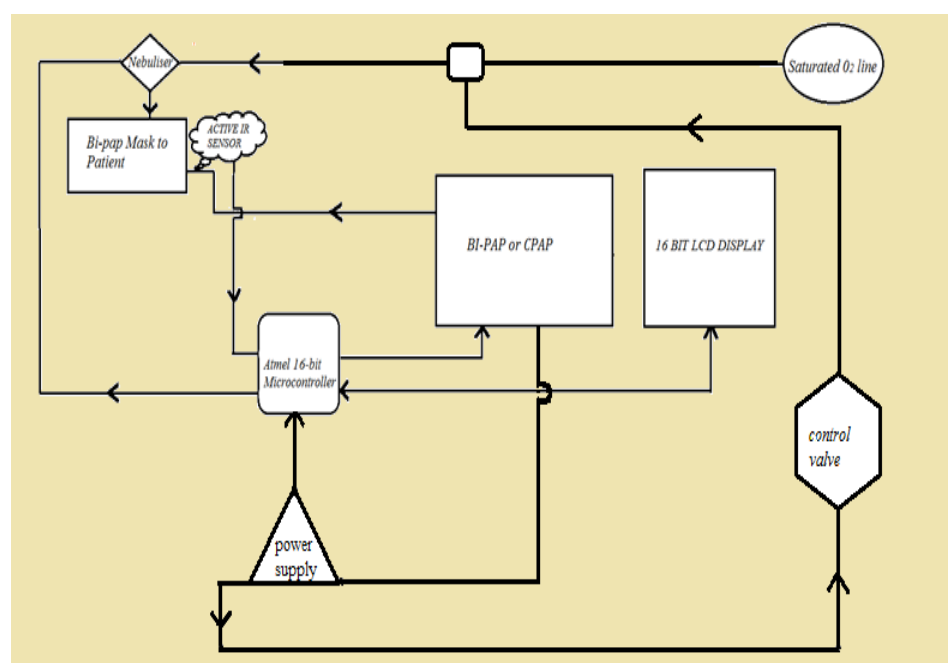

Figure 3.1: Design of Automatic-BiPAP

\subsection{Step by Step Working Procedure}

- Initially, the whole sleep apnea kit is placed on a table which is adjacent to the patient's sleeping bed.

- Just before he/she is about to sleep, he/she should wear the mask, tighten it with soft gloves and then switch ON the kit.

- Now, he/she should give the set value( i.e., 1,2) and then press enter key

1) Amount of time BiPAP should be in $\mathrm{ON}$ position (mins)

2) Sensor detection time $(10+/-3 \mathrm{secs})$ : as this time varies among adults and pediatrics

- When patient starts breathing, the fiber present in the mask moves accordingly up and down .This makes the IR sensor to sense the breathing cycle of a patient.

- For each breathing cycle detected by the sensor, there will be indication in display $(1 \rightarrow 0$ and $0 \rightarrow 1)$. This variation makes the counter to increment the number of breathing cycles per minute.

- The counter displays number of breathing cycles per minute on the LCD display. This count value reset to zero for every 60 seconds of time.

- This process continues whole night if the patient's breathing rate is normal or sleep apnea condition is absent.

If the patient's breathing rate is abnormal or sleep apnea condition is evolved.

- The IR sensor automatically checks the condition, that there should at least 1 breathing cycle within sensor detection time i.e., generally 10 secs.

- In case of sleep apnea patient, the above condition does not happens i.e., there will be pause in patient's breathing for around 10 secs.

- As soon as this situation arises, the sensor stops detecting respiratory rate and makes the BiPAP machine to switch ON eventually.

- Parallel to BiPAP machine, control valve is turned $\mathrm{ON}$ in order to pump saturated oxygen from humidifier to the patient's mask through oxygen line tubing with nebulizer kit (optional).

- This flow of positive air pressure continues, thereby making patients upper air way clear and the humidifier increases the moisture content in the trachea in order resolve many sleep disturbances like oral congestion etc.

- In case of central sleep apnea, a $24 \mathrm{v}$ AC buzzer is also activated in order to alert patient care taker around him.

- The BiPAP supplies air till the given set time, which is ensured through a switch on the kit (point 1) at the beginning.

- After the set time, The BiPAP machine automatically switches OFF consecutively the IR sensor starts sensing the breathing rate as usually.

- This process takes place accordingly whole night improving quality of sleep and supporting to patient's breathing undisturbed from sleep apnea event.

\subsection{Specific Operation of Each Component Used}

\subsubsection{Placement of IR Sensor in the Non-Invasive Mask}

The initial step of the design process was to select the optimal technique for sensing breathing rate of a patient. Generally, breathing rate of a healthy person ranges 12-18 breathing cycles/min. But it varies among infants and patients with respiratory abnormalities. Inside the mask, there will be thin, weight less plate made up of fiber or rubber like material as shown in fig 3.2. This plate allows inspired air to pass through it, while it closes eventually when expiration takes place. The active infrared source and detector is place diagonally on the opposite ends of the fiber plate as shown in fig 3.2.

The IR source sensor will be always delivering light radiation and is collected on the other end by the IR detector sensor (generally black colored). For every movement of the fiber, (i.e., for every inspiration and expiration cycle) the continuous radiation between IR source and detector is disturbed .This each and every disturbance is counted as single breathing cycle. This disturbance in radiation ensures breathing rate and is displayed on 16-bit LCD display. 


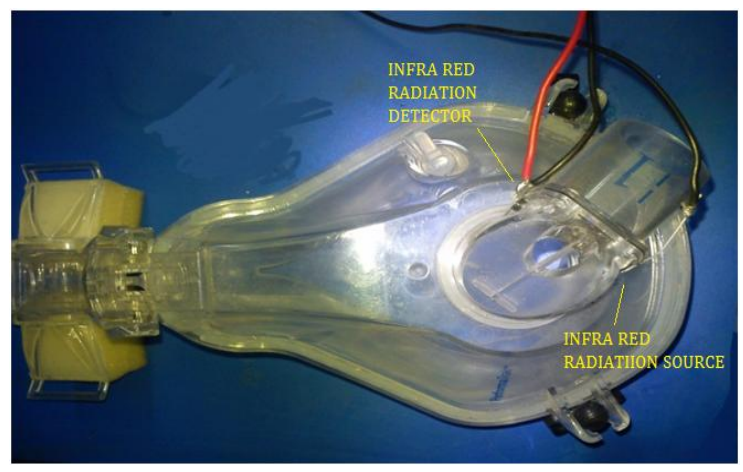

Fig 3.2. Placement of sensor in the mask

\subsubsection{Components Attached to the Non-Invasive Mask.}

In addition to the Active IR Sensors attached to the mask,

- The mask is assembled with long BiPAP tubing in order to pump positive air pressure supply.

- Thin Oxygen line tubing is attached to a inbuilt passage on the upper surface of the mask. This tubing is managed through a humidifier circuit.

- Optionally, a small nebulisation kit can be assembled to the oxygen tubing line.

\subsection{Pcb Circuit Explanation}

The entire printed circuit board is neatly attached to fiber switch board through screw system, which can be easily detachable and to follow safety precautions. The components in PCB are connected as shown in above schematic diagram. The PCB is powered through a 230V 3-pin power cord. Two Step-down transformers are used to regulate output AC voltage as 9-0-9V and $24 \mathrm{~V}$ respectively This $9-0-9 \mathrm{~V}$ is given to a forward bias 1n4007 diode which acts as rectifier Converting AC to DC. The rectified DC output from the diode is passed through the capacitor $(1000 \mathrm{uF}, 25 \mathrm{~V})$ for filtering, where ripples are eliminated. This filtered output is given to LM 7805 Regulator which gives constant voltage of 5 volts. This $5 \mathrm{~V}$ is used to switch IC ports between 1 and 0 accordingly.

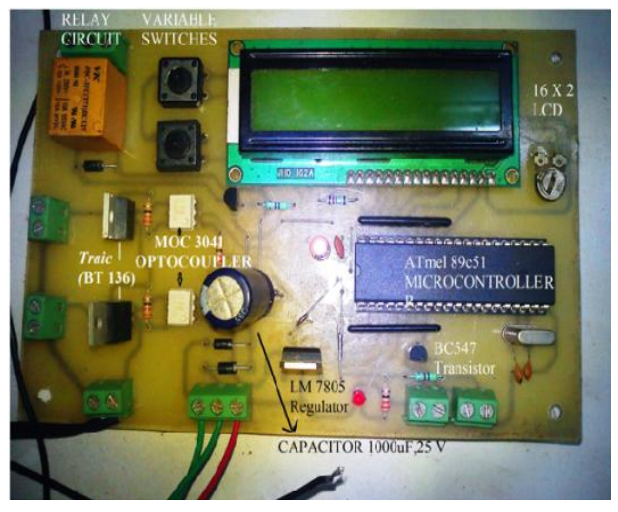

Fig 3.3 PCB circuit front schematic view
The other transformer which maintains $24 \mathrm{~V}$ output is specifically used for controlling relays and opto-coupler circuit. This opto-coupler circuit controls the BI-PAP i.e., when output voltage from transformer is given to opto-coupler circuit MOC3041, it drives the Triac (BT 136) This MOC device consist of gallium arsenide infrared emitting diodes optically coupled to a monolithic silicon detector performing the function of a Zero Voltage Crossing bilateral triac driver. They are designed for use with a triac in the interface of logic systems. As soon as Triac is driven, it conducts 24V AC to switch ON/OFF electro-controller valves.

\subsection{Control Switch Operated By Microcontroller}

As microcontroller is powered through a LM7805 regulator which allows only 5 volts to perform accordingly. This $5 \mathrm{~V}$ enables the IC ports 0 and 1 on the microcontroller. The 2 keys on the keyboard i.e., ground and micro-port helps to set time at which micro-controller has to perform. One key is connected to ground where as the other key works as enter button to activate microcontroller. When there is logic low or high in microcontroller, then accordingly the program gives the output.

\subsection{Timer Control}

The Microcontroller used here controls the timer circuit. For every output from IR sensor (i.e., for every inspiration and expiration by the patient, $1 \rightarrow 0$ and $0 \rightarrow 1$ respectively), the controller counts as ' 1 ' breathing cycle and is displayed on LCD display as shown in fig.3.3. Parallel to this event, the digital clock starts counting time in terms of seconds and minutes. For every breathing cycle there will be incremental counter manipulating number of breathing cycles per minute. If patient's breathing is normal (i.e., 12- 18 breath cycles/min), then IR sensor continues to sense patients breathing cycle progressively and displays number of breathing cycles per min.

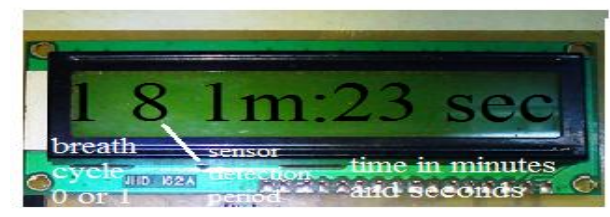

Fig 3.4 LCD display Showing breath cycles per minute

\subsection{Bi Pap Control Operations}

In this Hardware design of Automatic BiPAP machine, the power will be always delivering to the BiPAP machine. But, the BiPAP will be in position that it does not supply any positive air pressure to the patient's mask. The supply of positive air pressure is controlled by Microcontroller and a relay circuit in PCB.

The Micro controller is programmed as described below to control BiPAP operation: 
- Initially, The BiPAP machine will be in "CALM" position: when patient's breathing is Normal i.e., 12 18 breathing cycles/minute. In this position, The Active IR Sensors will be switch "ON" and counting will be as usual.

- Secondly, when the patients breathing is out of range (i.e. when breathing rate is out of range, abnormal For example, In case of sleep apnea patients pause in breathing is around more than $10 \mathrm{sec}$ ). Then, BiPAP machine turns on into "ACTIVE" position. In this Active position, the BiPAP machine will start pumping positive air pressure to the patients. In contrast to the above position, The Active IR sensors will be in switch "OFF" position. This OFF position continues for certain set time which can be adjustable manually.

\subsection{Regulation of Oxygen through Oxygen Line.}

The oxygen delivering to the patients mask is controlled by a "Microcontroller" and there by a "Control valve". When the sleep apnea patient is in normal condition there is no need of oxygen or BiPAP machine. If there is controversy condition, sometimes there is necessity of oxygen along with Positive Air Pressure from the machine. Here, Supply of oxygen to the patient maintains the level of oxygen concentration in patient's body and helps in early recovery.

Microcontroller is programmed in such a way that, the oxygen starts delivering to the patient: When abnormality condition is found by the sensors, BiPAP is turned ON there by Control Valve is opened in parallel with BiPAP.

\subsection{Humidifier}

When a patient is requiring mechanical ventilation over a longer period of time, it is imperative to take measures to compensate the losses of heat and moisture in order to avoid the aforesaid complications. Respiratory gas humidification is a method of artificial warming, conditioning and humidifying of respiratory gas for the patient during mechanical ventilation i.e., BiPAP. These three essential functions of respiratory gas conditioning constitute the preparation of inspired respiratory gas for the sensitive lungs. If natural respiratory gas humidification fails, pulmonic infections and damaging of lung tissues may be the consequence.

In case of sleep apnea patient, the patient is subjected to prolonged BiPAP therapy. Under this condition, continuous passage of dry air (low moisture content) into the patients air way. For example, in cities like Agra, Bhopal etc the amount of moisture content in atmosphere is less. So, there is a need of humidifier along with BiPAP machine. In addition to that, concentration of oxygen level reduces in sleep apnea patient because of irregular breathing intervals. Following to these circumstances sleep apnea patients requires humidifier for easy assistance in breathing. Fig below shows humidifier used in this research.

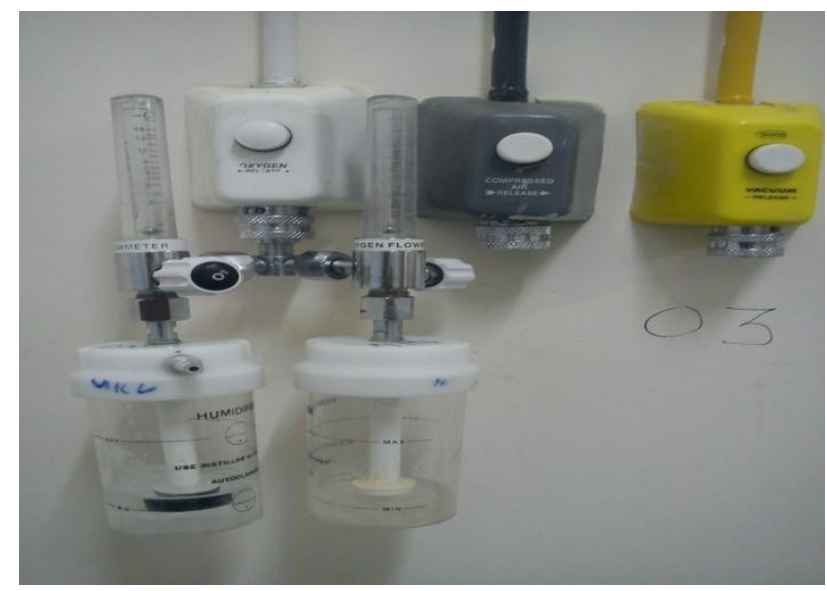

Fig 3.5 Shows Humidifier Circuit for Saturation

The control valve assembled to the humidifier turns ON, when BiPAP machine is turned ON. This allows the Humidified Oxygen to pass through the patients mask. The control valve closes after certain interval depending on patient's breathing cycle.

\subsection{Nebulisation Kit}

A small optional Nebulising device can be assembled in the oxygen line connected from humidifier to the patient's mask. Generally, Nebulizers are used for delivery of inhaled medications since they transform a liquid medication into a mist that can be comfortably and easily inhaled by a patient. The mist consists of a suspension of many miniscule liquid droplets in air and is created by the nebulizer rapidly, forcibly, and repeatedly disrupting the surface tension of the water and throwing droplets from the bulk liquid surface into the air. Depending on the level of breathing assistance necessary to counteract the airway closure and constriction of the trachea in patients with sleep apnea and asthma, certain amount ( 3 to $6 \mathrm{ml}$ ) of medication (bronchodilator) is given in reservoir of nebulizer for every prescribed time interval.

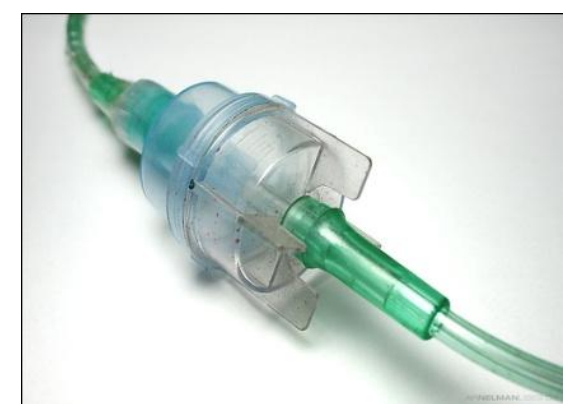

Fig.3.6. Nebulisation Chamber. 


\subsection{Buzzer or Beeper}

An electromechanical buzzer (audio signaling device) used in this research works on $24 \mathrm{v}$ AC supply. This buzzer can be optional depending on type of sleep apnea patient. Generally, this buzzer is used for central sleep apnea patients (as their brain does not send signals that regulate abnormal breathing).This activates in parallel with BiPAP machine there by alerting patient care takers to take remedies. Fig.3.7 shows a replicate image of buzzer.

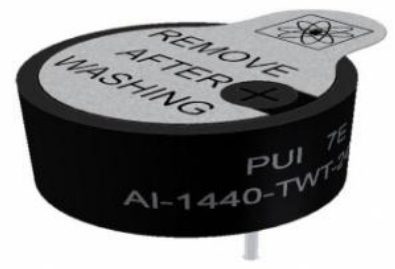

Fig.3.7 Replicate of Buzzer Image Used In PCB

\section{RESULTS AND CONCLUSION}

\subsection{Results}

\subsubsection{Ideal PCB Display}

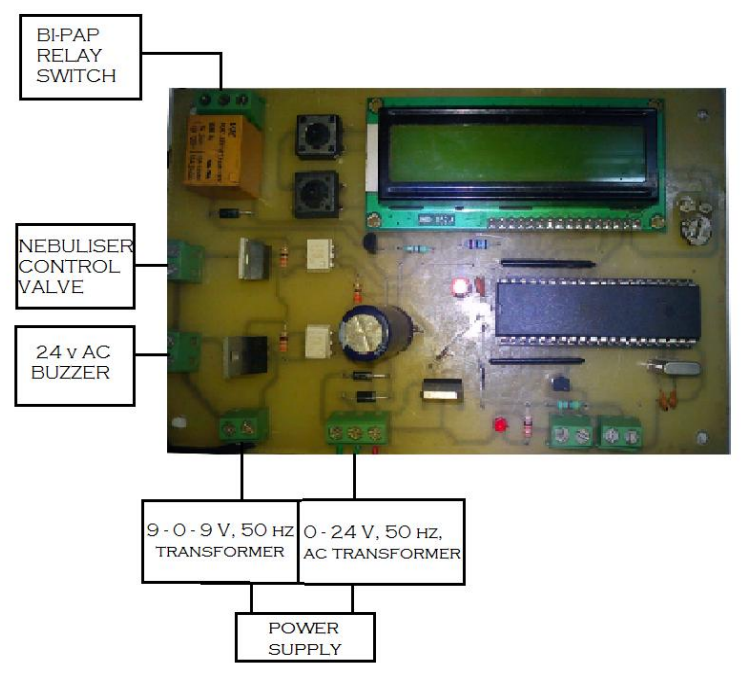

Fig. 4.1 Block Diagram of Ideal Working Model BiPAP

Fig 4.1 is an image of a working model which shows an ideal display prior to switch $\mathrm{ON}$ the circuit. Once power is supplied through these two transformers (9-0-9 v \& 0-24 v) and BiPAP device, the hardware starts displaying as shown in figure 4.2. In parallel to that, the BiPAP turns ON in Calm Mode (does not supply positive air pressure). The additional features like Relay circuit, Nebulizer Control valve and buzzer activates depending on the necessity of patient.

\subsubsection{Initialization of Sleep Apnea Kit}

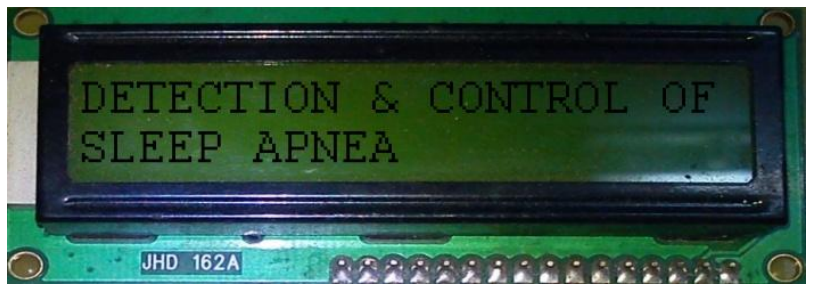

Fig 4.2 Display Showing Aim Of This Research Design

As soon as power is supplied to the PCB circuit, it starts displaying the title of the research "DETECTION AND CONTROL OF SLEEP APNEA THROUGH AUTOMATIC BIPAP" as shown in fig 4.2. Mask is connected firmly to the patient who is about to sleep or lay down. The control switches in the PCB should be manipulated to adjust the basic settings.

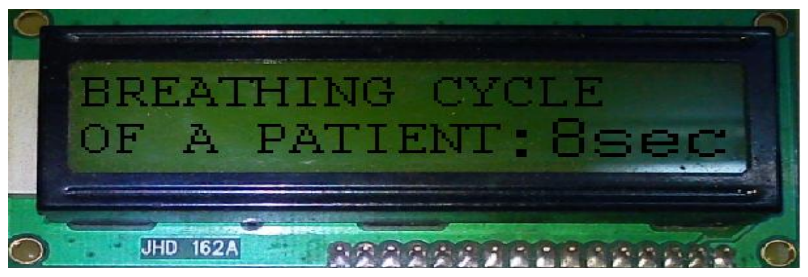

Fig 4.3 Manipulating Control Switch According To Patients Breathing Cycle

Depending on type of patient (adult or infant), type of disease (snoring, OSA, CSA) breathing cycle varies among individuals. According to that analysis, breathing cycle of a patient should be adjusted in the PCB manually. This can be varied in the interval $3 \operatorname{secs}$ to $12 \mathrm{secs}$ as per doctor suggestion. This breathing cycle is sensed by Infra Red Source and detector circuit.

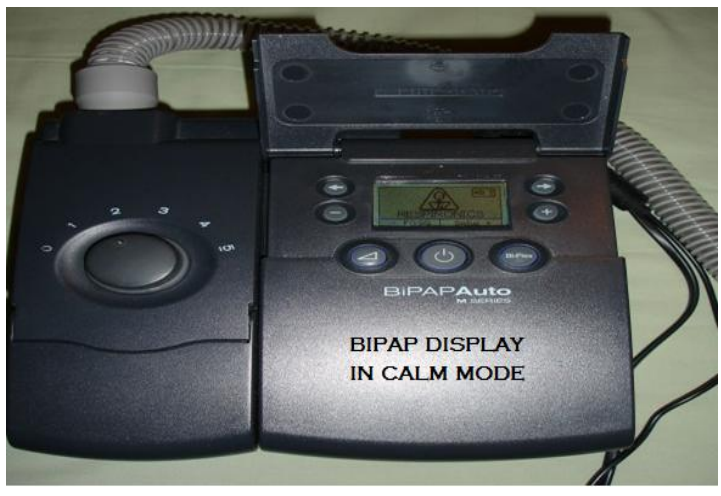

Fig 4.4 Display of BiPAP Machine in Calm Mode

BiPAP machine will be initially in calm mode as shown in figure 4.4. When the machine turns $\mathrm{ON}$, it starts delivering positive airway pressure with the settings adjusted in the previous session. 


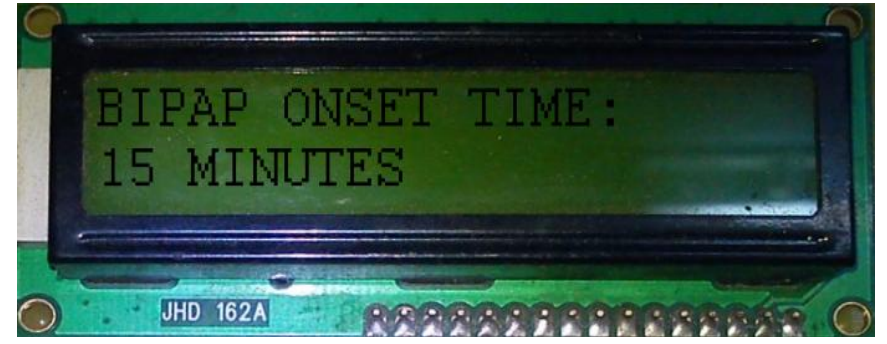

Fig 4.5 Manipulating Onset Time of a BiPAP Device

The amount of time the BiPAP should be in ON position will be decided by Micro-controller 89c51. This decision is followed as per the "ONSET Time" which is given manually as shown in fig 4.5. After supplying positive air way pressure up to specified onset time Bi-PAP switches back into calm mode. The onset time of a Bi-PAP is varied in terms of minutes and can be variable up to one hour.

\subsubsection{Sensor Acting on the Subject}

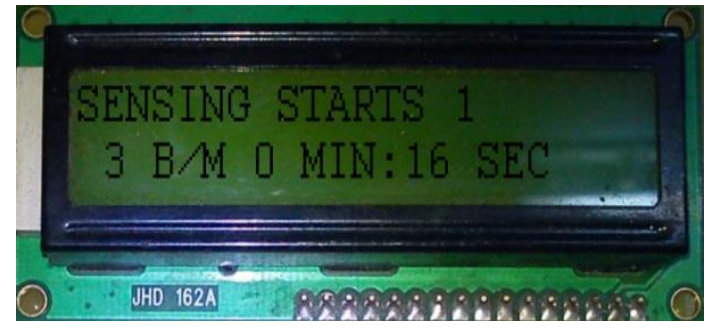

Fig.4.6 IR Sensor Sensing the Breathing Cycle of a Patient

The above image indicates number of breathing cycles patient is exchanging per minute. In detail IR sensor counts every inspiration and expiration in terms of 1's and 0's respectively as shown in the first row of the display. Second row is displaying number of breathing cycles he/she takes eventually i.e., 3 breathing cycles with in a time period of 16 seconds here.

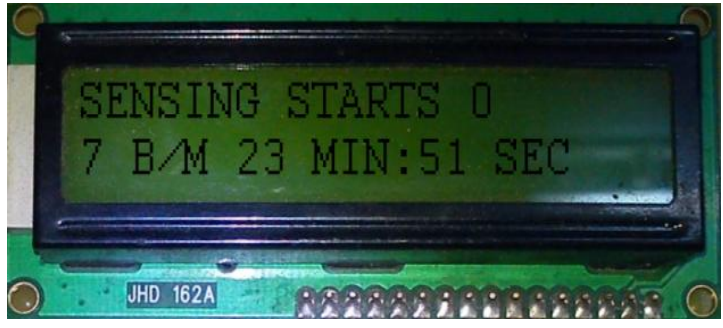

Fig 4.7 Sensing Abnormalities in Breathing

Here, we can understand that patient's breathing condition is abnormal i.e., Apnea condition is evolved displaying only seven breathing cycles for a time interval of 51 seconds. This situation may create inconvenience to his/her sleep and sometimes may cause life threatening effects. So, there is necessity of BiPAP machine which widens patients' upper airway.

\subsubsection{BiPAP Supporting Patient's Respiration after} Detecting Apnea

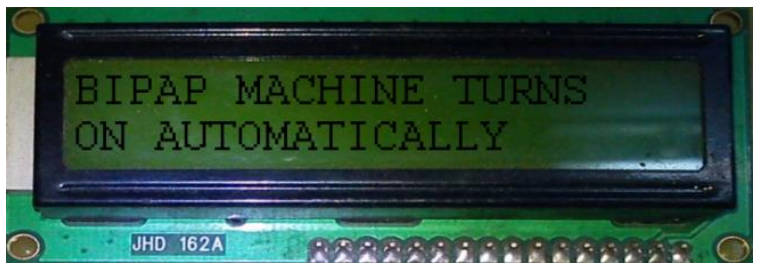

Fig 4.8 Display Showing That BiPAP Switched On Automatically

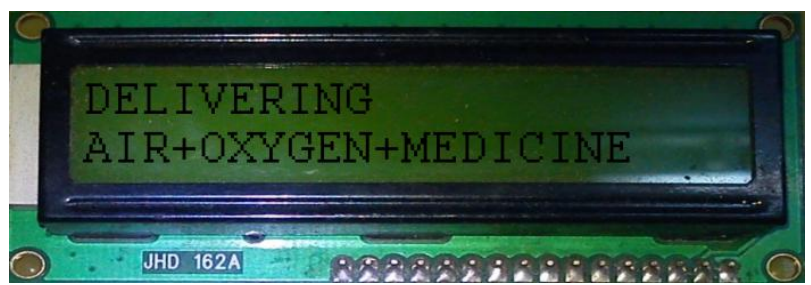

Fig 4.9 BiPAP Display Showing That Positive Air Pressure is Delivering to Patient

As soon as sleep apnea condition is evolved in the patients breathing cycle, BiPAP machine will be turned ON automatically by the Microcontroller without delay giving supportive positive air pressure to make patients upper airway open strictly. As shown in figure 4.9, along with positive air, saturated oxygen is also delivered directly into the patients mask with small tubing from oxygen humidifier. Optionally, a Nebulising kit can also be connected, if the patient has asthma along with apnea condition.

\subsubsection{Pressurized Air Controlling Effect of Apnea}

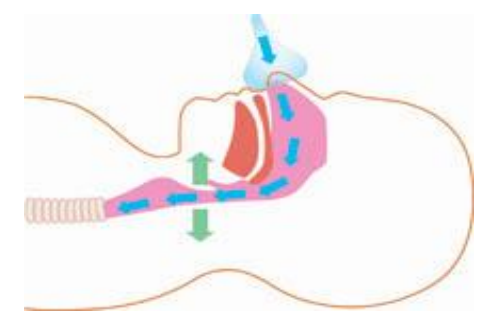

Fig 4.10 Shows Widening Trachea (not original)

When the BiPAP is turned on it pushes the upper airway such that it widens as shown in figure above. The humidified saturated oxygen along with this positive pressurized air helps out in increasing the moisture content in the trachea in order resolve many sleep disturbances like oral congestion etc. Along with this a nebulisation kit is also connected in order to clear airway of patients having both asthma and apnea. 

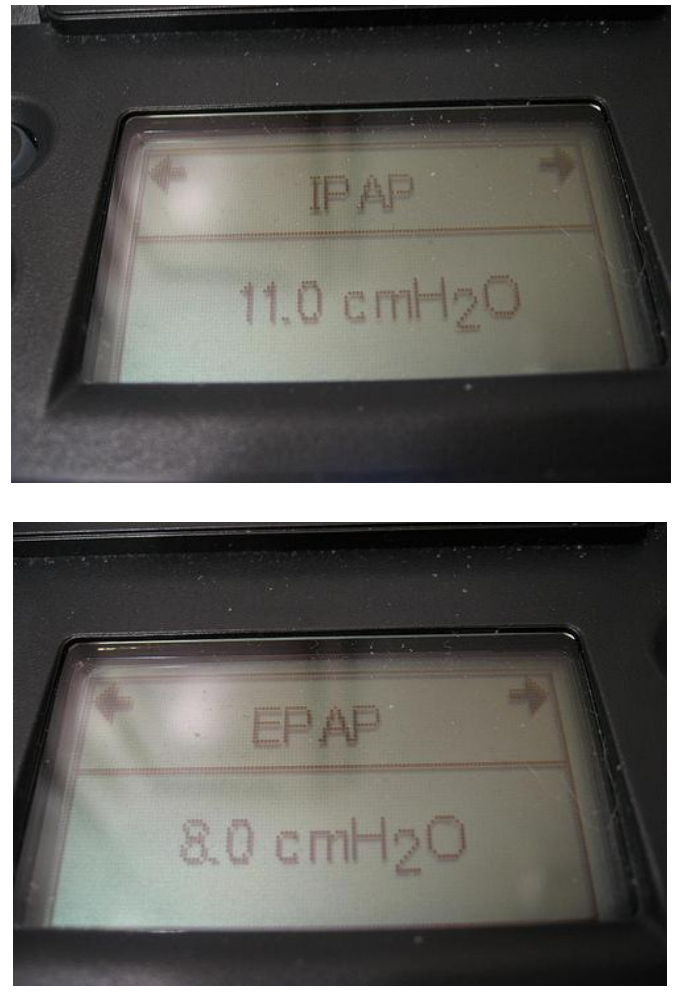

Fig 4.11 BiPAP Display Showing Inspiration and Expiration Volumes

The inspiration and expiration volumes delivering through BiPAP device are pre-set values i.e., these $\mathrm{cmH}_{2} 0$ volumes were the default values adjusted in the previous session of usage. These default values changes from patient to patient and are prescribed by physician. Now patient gradually feels comfort in taking breath in his/her sleep.

\subsubsection{Sensing Kit Following Cyclic Process Whole}

\section{Night}

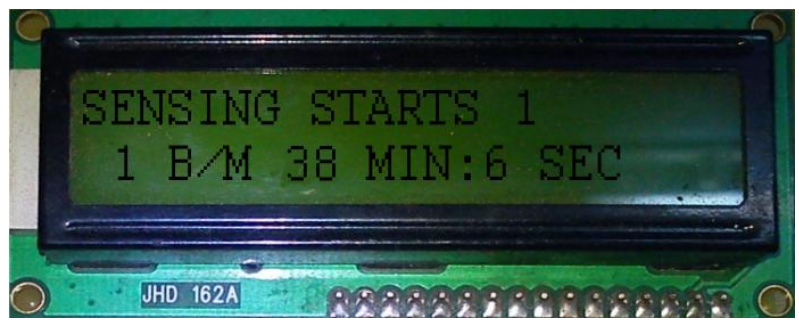

Fig.4.12 Apnea Sensing Starts Again After Certain Time

After certain interval of time (BiPAP Onset time), irrespective of patients breathing condition BiPAP machine turns OFF and senor gets activated. There by, sensing starts again to check apnea condition of a patient. By this activity, the Amount of positive air delivering by a BiPAP machine can be reduced (without using device for the whole night).
Along with this Effect, a 24v AC buzzer is also connected to the PCB circuit, which can alert the patient care takers in case of central sleep apnea. This buzzer can be optional setting depending on type of patient. Thus, the obtained outcomes from the current design give an idea about how entire hardware is sophisticated in detection and control of sleep apnea disease.

\subsection{Comparison between Current and Existing Bi-PAP} Designs

\begin{tabular}{|c|c|c|}
\hline & CURRENT DESIGN & EXISTING DESIGN \\
\hline COST & $\begin{array}{lr}\text { Economically } & \text { good } \\
\text { compared to } & \text { Existing } \\
\text { designs } & \text { (Rs9000 } \\
\text { approx) } & \\
\end{array}$ & $\begin{array}{l}\text { Present day Basic Bi- } \\
\text { PAP machines costs } \\
\text { more than Rs } 80,000\end{array}$ \\
\hline $\begin{array}{l}\text { DISPLAY } \\
\text { SYSTEM }\end{array}$ & $\begin{array}{l}\text { The Display system is } \\
\text { user free, which can be } \\
\text { easily understandable } \\
\text { by common people }\end{array}$ & $\begin{array}{l}\text { The display system is } \\
\text { complex, which cannot } \\
\text { be understandable }\end{array}$ \\
\hline USAGE & $\begin{array}{l}\text { This device is } \\
\text { compatible for both } \\
\text { home and hospital } \\
\text { usage }\end{array}$ & $\begin{array}{l}\text { This device is mostly } \\
\text { used in hospitals as the } \\
\text { settings cannot be } \\
\text { easily understood by a } \\
\text { common person. }\end{array}$ \\
\hline $\begin{array}{l}\text { STERILIZ } \\
\text { ATION }\end{array}$ & $\begin{array}{l}\text { Apnea Sensing } \\
\text { components can be } \\
\text { easily detachable } \\
\text { before sterilization }\end{array}$ & $\begin{array}{l}\text { Sensing components } \\
\text { are not present outside } \\
\text { the circuit }\end{array}$ \\
\hline SETTINGS & $\begin{array}{l}\text { Depending on infants } \\
\text { or adults breathing } \\
\text { cycle, settings can be } \\
\text { adjusted just by using } \\
\text { two switches. }\end{array}$ & $\begin{array}{l}\text { Complexity in } \\
\text { adjustment of settings } \\
\text { may cause usage of }\end{array}$ \\
\hline $\begin{array}{l}\text { LOGIC } \\
\text { USED }\end{array}$ & $\begin{array}{l}\text { Simpler Infra red } \\
\text { radiation source and } \\
\text { detector is used, which } \\
\text { is cheaper and } \\
\text { accurate }\end{array}$ & $\begin{array}{l}\text { Generally, Flow sensor } \\
\text { or pressure sensor is } \\
\text { used, which is cost } \\
\text { effective }\end{array}$ \\
\hline $\begin{array}{l}\text { BUZZER / } \\
\text { BEEPER }\end{array}$ & $\begin{array}{l}\text { Depending on type of } \\
\text { patient i.e., OSA or } \\
\text { CSA, Buzzer can be } \\
\text { turned ON/OFF by } \\
\text { examining Sleep apnea } \\
\text { condition. }\end{array}$ & $\begin{array}{l}\text { Irrespective of the } \\
\text { patient type, buzzer } \\
\text { activates disturbing the } \\
\text { patients sleep. Alerts } \\
\text { only if there is leak. }\end{array}$ \\
\hline $\begin{array}{l}\text { NEBULIZE } \\
\mathrm{R}\end{array}$ & $\begin{array}{l}\text { Along with positive } \\
\text { airway pressure, } \\
\text { nebulizer can also be } \\
\text { connected such that it } \\
\text { clears patients blocked } \\
\text { airway with } \\
\text { bronchodilators } \\
\text { (generally in case of } \\
\text { patients with sleep } \\
\text { apnea and asthma) }\end{array}$ & $\begin{array}{l}\text { Only BiPAP is } \\
\text { engaged. it doesn't } \\
\text { support nebulizer }\end{array}$ \\
\hline
\end{tabular}




\subsection{Advantageous Additional Features Included In the Current Design}

$>$ The Infra Red radiation emitter and detector sensing system can easily detect the number of breathing cycles of the subject for a certain interval of time and is displayed.

$>$ BiPAP machine can turn ON in required intervals of sleep depending on patient's respiratory track.

$>$ The humidification circuit is auto-controlled by a control relay which activates along with BiPAP device such that patient's trachea does not undergo congestion (in comfort).

$>$ In this design, Exclusively Central sleep apnea patients are engaged with a buzzer such that their abnormal condition will be intimated as alarm signal to the patient care takers.

$>$ In this design, as the BiPAP "On-time" is very less, power consumption will be low, which is an advantageous feature in this research.

\subsection{A Patient Report in Mallya Hospital, Bangalore}

\begin{tabular}{|c|c|c|c|c|c|}
\hline \multicolumn{6}{|c|}{ NAME OF THE PATIENT : Mrs. BANGARAPPA } \\
\hline AGE: 65 & $\begin{array}{l}\text { SEX: } \\
\text { F }\end{array}$ & \multicolumn{2}{|c|}{$\begin{array}{l}\text { WEIGHT : } 78.0 \\
\mathrm{~kg}\end{array}$} & \multicolumn{2}{|c|}{$\begin{array}{l}\text { DATE : 29-05- } \\
2011\end{array}$} \\
\hline \multicolumn{6}{|c|}{ DISEASE : Congestion in Breathing, Sleep Apnea } \\
\hline \multicolumn{2}{|c|}{$\begin{array}{l}\text { DEVICE TURN ON } \\
\text { TIME }\end{array}$} & \multicolumn{4}{|c|}{ DEVICE TURN OFF TIME } \\
\hline \multicolumn{2}{|c|}{$\begin{array}{l}\text { Day 1 }=7: 10 \mathrm{pm} \\
\text { Day 2 }=8: 56 \mathrm{pm}\end{array}$} & \multicolumn{4}{|c|}{$\begin{array}{c}8: 16 \mathrm{pm} \\
10: 05 \mathrm{pm}\end{array}$} \\
\hline \multicolumn{6}{|c|}{ 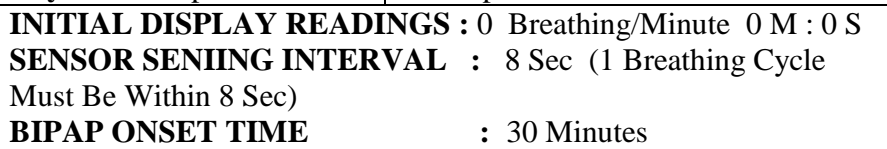 } \\
\hline $\begin{array}{l}\text { Device Running } \\
\text { Interval (Day 1) }\end{array}$ & \multicolumn{2}{|c|}{$\begin{array}{l}\text { Breathing } \\
\text { Cycles Per } \\
\text { Min }\end{array}$} & \multicolumn{2}{|c|}{$\begin{array}{l}\text { Device Running } \\
\text { Interval (Day 2) }\end{array}$} & $\begin{array}{l}\text { Breathing } \\
\text { Cycles Per } \\
\text { Minute }\end{array}$ \\
\hline 7:11 Pm (1 Min) & \multicolumn{2}{|c|}{14} & \multicolumn{2}{|l|}{$8: 56$ (1 Min) } & 12 \\
\hline 7:12 Pm (2 Min) & \multicolumn{2}{|l|}{13} & \multicolumn{2}{|l|}{$8: 57$ (2 Min) } & 14 \\
\hline 7:13 Pm (3 Min) & \multicolumn{2}{|l|}{11} & \multicolumn{2}{|l|}{$8: 58$ (3 Min) } & 11 \\
\hline 7:14 Pm (4 Min) & \multicolumn{2}{|l|}{16} & \multicolumn{2}{|l|}{$8: 59$ (4 Min) } & 13 \\
\hline $7: 15 \mathrm{Pm}$ (5 Min) & \multicolumn{2}{|l|}{13} & \multicolumn{2}{|l|}{ 9:00 (5 Min) } & 11 \\
\hline 7:16 Pm (6 Min) & \multicolumn{2}{|l|}{12} & \multicolumn{2}{|l|}{ 9:01 (6 Min) } & 12 \\
\hline 7:17 Pm (7 Min) & \multicolumn{2}{|l|}{11} & \multicolumn{2}{|l|}{ 9:02 (7 Min) } & 15 \\
\hline $7: 18 \mathrm{Pm}(8 \mathrm{Min})$ & \multicolumn{2}{|l|}{14} & \multicolumn{2}{|l|}{ 9:03 (8 Min) } & 13 \\
\hline 7:19 Pm (9 Min) & \multicolumn{2}{|l|}{13} & \multicolumn{2}{|l|}{ 9:04 (9 Min) } & 14 \\
\hline 7:20 Pm (10 Min) & \multicolumn{2}{|l|}{11} & \multicolumn{2}{|c|}{ 9:05 (10 Min) } & 13 \\
\hline 7:21 Pm (11 Min) & \multicolumn{2}{|l|}{12} & \multicolumn{2}{|c|}{ 9:06 (11 Min) } & 14 \\
\hline 7:22 Pm (12 Min) & \multicolumn{2}{|l|}{11} & \multicolumn{2}{|c|}{ 9:07 (12 Min) } & 13 \\
\hline 7:23 Pm (13 Min) & 8 & & 9:08 (13 Mil & & 15 \\
\hline 7:55 Pm (1 Min) & 14 & & 9:09 (14 Mi & & 10 \\
\hline
\end{tabular}

\begin{tabular}{|c|c|c|c|}
\hline 7:56 Pm (2 Min) & 13 & 9:10 (15 Min) & 15 \\
\hline 7:57 Pm (3 Min) & 14 & 9:11 (16 Min) & 13 \\
\hline 7:58 Pm (4 Min) & 12 & 9:12 (17 Min) & 11 \\
\hline 7:59 Pm (5 Min) & 13 & 9:13 (18 Min) & 16 \\
\hline 8:00 Pm (6 Min) & 11 & 9:14 (19 Min) & 15 \\
\hline 8:01 Pm (7 Min) & 15 & 9:15 (20 Min) & 09 \\
\hline 8:02 Pm (8 Min) & 14 & 9:16 (21 Min) & 15 \\
\hline 8:03 Pm (9 Min) & 10 & 9:17 (22 Min) & 14 \\
\hline 8:04 Pm (10 Min) & 13 & 9:18 (23 Min) & 12 \\
\hline 8:05 Pm (11 Min) & 13 & 9:19 (24 Min) & 07 \\
\hline 8:06 Pm (12 Min) & 14 & 9:50 (1 Min) & 13 \\
\hline 8:07 Pm (13 Min) & 12 & 9:51 (2 Min) & 14 \\
\hline 8:08 Pm (14 Min) & 11 & 9:52 (3 Min) & 11 \\
\hline 8:09 Pm (15 Min) & 13 & 9:53 (4 Min) & 13 \\
\hline $8: 10$ Pm (16 Min) & 14 & 9:54 (5 Min) & 12 \\
\hline $8: 15$ PM (17 Min) & 13 & 9:55 (6 Min) & 14 \\
\hline & & 9:56 (7 Min) & 13 \\
\hline & & 9:57 (8 Min) & 13 \\
\hline & & 9:58 (9 Min) & 11 \\
\hline & & 9:59 (10 Min) & 16 \\
\hline & & 10:00 (11 Min) & 13 \\
\hline & & 10:01 (12 Min) & 12 \\
\hline & & 10:02 (13 Min) & 15 \\
\hline & & 10:03 (14 Min) & 13 \\
\hline & & 10:04 (15 Min) & 12 \\
\hline \multicolumn{4}{|c|}{$\begin{array}{l}\text { REMARKS: At 7:24 PM (day 1) \& 9:20 (day } 2) \text {, the breaths } \\
\text { didn't reach the normal range }(12-18 \mathrm{~b} / \mathrm{m}) \text {. So, the device } \\
\text { starts giving positive pressure in order to compensate the } \\
\text { abnormal condition. This Machine delivers continuously for a } \\
\text { certain time interval ( } 30 \text { mins). After this time interval sensor } \\
\text { again starts sensing as usual in cyclic process. }\end{array}$} \\
\hline
\end{tabular}

\section{CONCLUSIONS}

Finally, it is concluded that this fully assembled Bi-PAP is advantageous for people belonging to rural and resource-less people. This is compatible for both home and hospital usage. Easily understandable display is expedient in this equipment. The sleep state of a patient will not be disturbed as the Bi-PAP functioning depends on the patient's necessity for positive air pressure; there by the Bi-PAP acting time on a patient can be reduced and hence, BiPAP side effects can be minimized.Central sleep apnea can also be detected and alarmed such that this ability could prove critic when used during emergency situations as well as convenient when used for a sleeping patient. It is economically good and maintenance free. This process takes 
place accordingly whole night improving quality of sleep and supporting to patient's breathing undisturbed from sleep apnea event. However, further testing on sleep apnea patients is necessary to determine the true value of efficiency of this system.

\section{FUTURE SCOPE}

Significant progress has been accomplished on the BIPAP system during the design period. The device is in working condition and testing has been performed to validate the efficiency of the system. Based on the results of the testing, the Automatic BiPAP system was shown to be more efficient at detecting sleep apnea condition and responding to the patients breathing cycle accordingly

Slight modifications to the device could be implemented to improve the ease of use of the device in future. Modifications include the detection of volumetric respiratory rate by a flow sensor, thereby BiPAP acts accordingly. Additionally, improvements could be made to the user interface such that the breathing rate of a sleep apnea patient could be stored for further usage.The portability and display has to be improved. The system has to be validated for use on live subjects in the Sleep Research Laboratory.

\section{REFERENCES}

[1]. Sleep Apnea Association (ASAA). [Online]- Estes, M. C., J. Fiore, D. M. Mecklenburg, H. Ressler, and J. Kepler : www.sleepapnea.org, USA

[2].Delivery of Aerosolized Medication through Continuous Positive Airway Pressure Device- Professor John Webster, University of Wisconsin, Madison- BME 402, Bio-medical engineering design

[3].Home monitoring of sleep apnea treatment: benefits of intelligent CPAP devices -Daniela Boisteanu, Raluca Vasiluta, Andrei Cernomaz, Corina Mucenica 2009 - University of Medicine and Pharmacy "Gr.T.Popa" Iasi,Clinic of Pulmonary Diseases, Iaşi-2009 IEEE,10.11.09/AT-EQUAL Advanced Technologies for Enhanced Quality of Life.2009(pp77-80).

[4]. Long-term performance evaluation of an automatic air way positive pressured device- Fu-Chung Yen, M.S., Khosrow Behbehani, Ph.D., John R. Burk, MD.,Edgar A. Lucas, Ph.D., and John Axe, Ph.D.- 18th Annual International Conference of the IEEE Engineering in Medicine and Biology Society, Amsterdam 1996 (pp2113-2114)

[5]. Auto-CPAP (APAP) for Treatment of Obstructive Sleep Apnea- Richard B. Berry, M.D.Malcolm Randall, Department of Medicine, University of Florida -Proceedings of the Second joint EMBS/BMES Conference, Houston. TX. JSA * October 23-2002 (pp1542-1543)

[6]. Treatment apparatus employing air-bag actuation for sleep apnea syndrome- Shunji Moromugi, Takao Ayuse, Taichi Sakamoto, Yusuke Tomimatsu and Takakazu Ishimatsu, Nagasaki University-International Conference on Control, Automation and Systems 2008
Oct. 14-17, 2008 in COEX, Seoul, Korea (pp2010-2013)

[7]. Detection of Obstructive Sleep Apnea from Cardiac Interbeat Interval Time Series

JE Mietus, CK Peng, P Ch Ivanov, AL Goldberger -Beth Israel Deaconess Medical Center and Harvard Medical School, Boston, USA- IEEE 753 Computers in Cardiology 2000;(pp753-756).

[8]. Trial of Measurement of Sleep Apnea Syndrome with Sound Monitoring and $\mathrm{SpO} 2$ at home- Ashida Nobuyuki, Nasu Yasuhiro, Teshima Taik, Yamakawa Miyae, Makimoto Kiyoko and Higashi Terumasa- Dept. of Medical and Welfare Management, Koshien University, Takarazuka, JapanHealthcom 2009 IEEE (pg 66-69).

\section{WEBSITES}

1. www.sleepapnea.org

2. http://en.wikipedia.org/wiki/sleepapnea

3.http://www.nhlbi.nih.gov/health/dci/Diseases/SleepApnea/Sle epApnea_WhatIs.html

4. www.nlm.nih.gov/medlineplus/sleepapnea.html

5. http://www.medicinenet.com/sleep_apnea/article.html

6. http://www.helpguide.org/life/sleep_apnea.html

7. http://www.ninds.nih.gov/disorders/sleep_apnea/sleep_apnea .html

8._http://www.sleep-apnea-guide.com

9. http://www.sleepfoundation.org

10.http://www.umm.edu/patiented/articles/how_serious_sleep_a pnea_000065_5.html

\section{BIOGRAPHY}

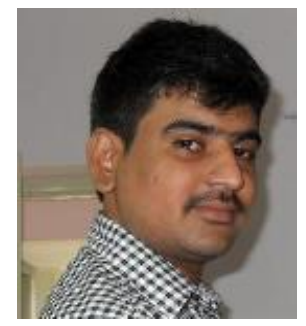

Sandeep Reddy AV is working as Assistant Professor in Gokaraju Rangaraju Institute of Engineering \& Technology. His areas of interest are Bio Medical, and he is working on same project to increase the features and to reduce the cost of the particular equipment. 